\title{
Evaluation of a Point-of-Care Ultrasound (POCUS) training course for Regional Anesthesiologists - A Single Institution's Experience
}

\author{
Alex K. Saltzman', Thuyvan H. Luu', Nicole Brunetti' ', James D. Beckman ${ }^{1,2}$, Mary J. Hargett', Stephen C. Haskins ${ }^{1,2 *}$ \\ 'Department of Anesthesiology, Critical Care \& Pain Management, Hospital for Special Surgery, New York, USA \\ ${ }^{2}$ Department of Anesthesiology, Weill Cornell Medicine, New York, USA
}

\section{Article Info}

\section{Article Notes}

Received: February 21, 2020

Accepted: June 01, 2020

\section{${ }^{*}$ Correspondence:}

*Dr. Stephen C. Haskins, Department of Anesthesiology, Critical Care \& Pain Management, Hospital for Special Surgery, New York, USA; Telephone: (212) 606-1036; Email: Haskinss@hss.edu.

${ }^{\circ} 2020$ Haskins SC. This article is distributed under the terms of the Creative Commons Attribution 4.0 International License.
Abstract

Background and Objectives: Point-of-care ultrasound (POCUS) in the form of focused cardiac ultrasound (FOCUS) is a powerful clinical tool for anesthesiologists to supplement bedside evaluation and optimize cardiopulmonary resuscitation in the perioperative setting. However, few courses are available to train physicians. At Hospital for Special Surgery (HSS), from March of 2013 to May of 2016, nine basic Focused Assessed Transthoracic Echocardiography (FATE) training courses were held. A large percentage of the participants were practicing regional anesthesiologists or trainees in fellowship for regional anesthesia and acute pain. In this study, a survey was used to assess clinical utilization as well as potential barriers to use for regional anesthesiologists.

Methods: Following IRB approval, 183 past participants of the basic FATE training course were contacted weekly from November $22^{\text {nd }}, 2016$, through January $3^{\text {rd }}, 2017$, via email and sent a maximum 40-item electronic survey hosted on REDCap. Responses were analyzed by a blinded statistician.

Results: 92 participants responded (50\%), and 65 of the 92 (70.7\%) indicated they had regional anesthesiology training or practice regional anesthesia regularly. Of the total number of respondents, $50 \%(95 \% \mathrm{Cl}$ : 40.3\%, 59.8\%; P-value $=0.001$ ) have used FOCUS to guide clinical decision making. Of the regional anesthesiologists, 27 (45.8\%) have used FOCUS to guide clinical decision making with left ventricular function assessment (40.7\%) and hypovolemia (39.0\%) being the most common reasons. Regional anesthesiologists utilized FOCUS in the following settings: preoperatively (44.6\%), intraoperatively (41.5\%), postoperatively (41.5\%), and in the Intensive Care Unit (40.0\%). Limitations were due to lack of opportunities (52.3\%), resources (36.9\%), and comfort with performance (30.8\%). $84.4 \%$ agreed that basic FOCUS training should be a required part of anesthesia residents or fellows' curriculum.

Conclusions: This study is the first formal evaluation of the impact of the implementation of a FOCUS training course on regional anesthesiologists' current practice. Nearly $50 \%$ of regional anesthesiologists used FOCUS to guide clinical decision-making following formal training. The limitations to the use of FOCUS were a lack of relevant opportunities and resources. This evaluation of clinical use following training provides insight into how FOCUS is used by regional anesthesiologists and the limitations to implementation in the perioperative setting.

\section{Introduction}

Point-of-care ultrasound (POCUS) in the form of focused cardiac ultrasound (FOCUS) and lung ultrasonography (LUS) is a powerful clinical tool for anesthesiologists to supplement clinical evaluation and optimize cardiopulmonary resuscitation in the perioperative setting ${ }^{1-3}$. FOCUS can detect significant changes in cardiac pathology, which can result in a change in perioperative management, 
including anesthetic or surgical plan ${ }^{1}$. For example, FOCUS and LUS can be used preoperatively to assess for significant valvular disease; intraoperatively to assess complications related to regional anesthesia such as hemodynamic instability, pneumothorax, and diaphragmatic paresis; and postoperatively to diagnose and manage potential complications $^{2-4}$. There is also a well-established role for POCUS in obstetric anesthesia 5 . Given that regional anesthesiologists routinely use ultrasound (US) in the form of ultrasound-guided regional anesthesia (UGRA), adding FOCUS and LUS to their skillsets can provide a cost-effective means to improve clinical management $t^{6}$.

In recognizing its growing importance, medical societies, including the Society of Critical Care Medicine ${ }^{7,8}$, American Society of Echocardiography ${ }^{9}$, the Society of Critical Care Anesthesiologists ${ }^{10}$, and the European Society of Paediatric and Neonatal Intensive Care (ESPNIC) ${ }^{11}$ have already created guidelines for various POCUS skills regarding clinical use and training programs. A series on POCUS in Regional Anesthesia and Pain Medicine (RAPM) has highlighted the role of LUS and FOCUS for the regional anesthesiologist and pain management specialist ${ }^{2-4}$. The American Society for Regional Anesthesia and Pain Management (ASRA) has also created an Introduction to Perioperative Point-
of-Care Ultrasound (POCUS) course that provides FOCUS training in the form of basic focus assessed transthoracic echocardiology (FATE) certification ${ }^{12}$. Despite its growing popularity, there are few standardized courses available to adequately train physicians on how to incorporate FOCUS and LUS into their daily practice ${ }^{13}$. The assessment of an anesthesia run peri-FOCUS service showed a significant alteration in the management of most patients regarding the guidance of fluid and vasoactive drugs administration and interventional percutaneous cardiac procedures ${ }^{14}$. Additionally, studies have shown that the implementation of training courses resulted in quick and effective training for residents ${ }^{15,16}$ and that medical students who complete formal training courses perceive point-of-care ultrasound training as beneficial ${ }^{17}$.

At Hospital for Special Surgery (HSS), nine basic FOCUS courses in the form of basic FATE have been held since 2013 as of November 2016. The goal of the basic FATE courses was to promote the incorporation of FOCUS into the routine practice of clinicians. The FATE Protocol is the oldest FOCUS protocol and provides a structured way to qualitatively assess the heart and pleura for obvious pathology ${ }^{18}$. Supplementary FATE protocol cards summarize the US views, which are helpful for novice users. (Figure 1) The

\section{regionmidtjylland midt}

\section{Focus Assessed Transthoracic Echo (FATE)}

Scanning through position 1-4 in the most favourable sequence
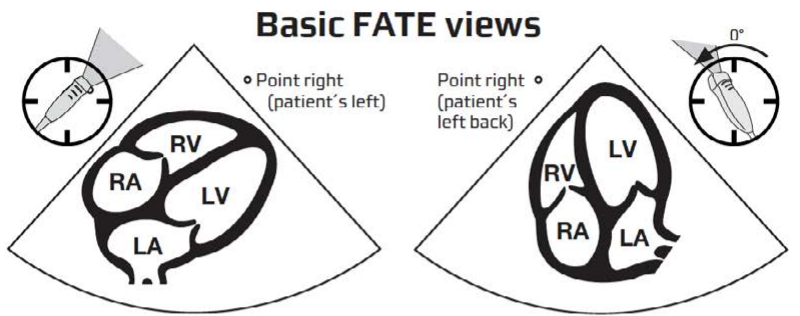

Pos 1: Subcostal 4-chamber

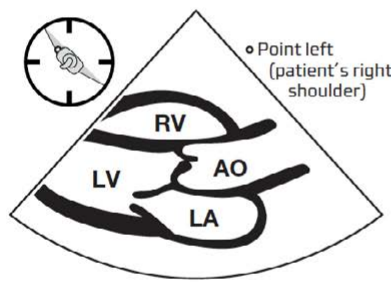

Pos 2: Apical 4-chamber

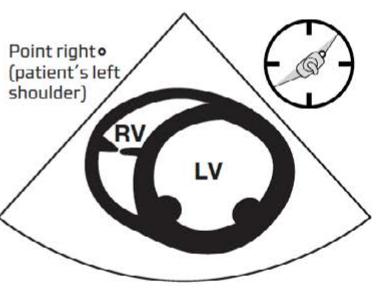

Pos 3: Parasternal LV short axis
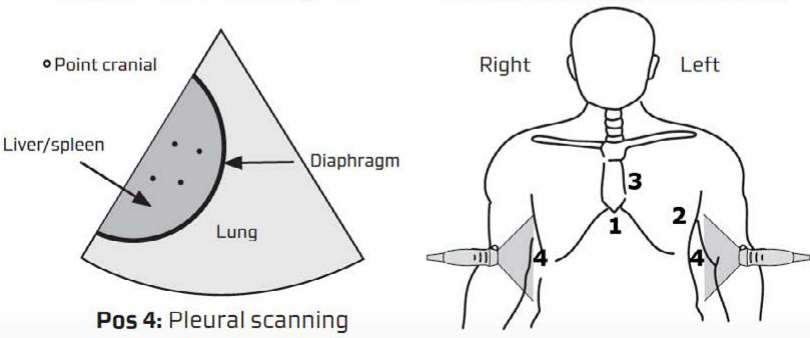

Figure 1: Focus Assessed Transthoracic Echocardiography (FATE) Card used as a supplement to the didactic and hands-on-training Focused Cardiac Ultrasound (FOCUS) courses. 
basic FATE course involves the completion of an online "e-learning" platform that teaches the fundamentals of FOCUS and LUS. The reverse classroom model allows for the learner to attend a one-day in-person course with six hours dedicated to hands-on-training on standardized models. The faculty teaching the hands-on portion of the course are chosen based on their experience performing and teaching transthoracic echocardiograph as well as having basic FATE certification. The goal of this course is to teach participants the basic FATE views (subcostal 4-chamber, subcostal IVC view, apical 4-chamber, parasternal long axis, parasternal short-axis views, and pleural imaging) and M-Mode assessment for Mitral Annular Plane Systolic Excursion (MAPSE) / Tricuspid Annular Plane Systolic Excursion (TAPSE), Mitral Septal Separation (MSS) and Fractional Shortening (FS). Another goal of the courses taught at HSS is to teach participants proper LUS technique, including patient positioning, probe selection, and placement, and a systematic approach. FOCUS and LUS training allow for assessment of potential complications related to regional anesthesia, such as hypovolemia and acute respiratory distress due to pneumothorax or hemidiaphragmatic paresis ${ }^{2-3}$. In addition to the courses at HSS, the FATE course is a formal taught by ASRA and yields a CME certification ${ }^{12}$.

In this study, a survey was used to assess how participants who completed the basic FATE course perceive the incorporation of FOCUS training in their practice, determine the potential limitations to FOCUS utilization, and assess the benefits of their FOCUS training ${ }^{19}$. Specifically, we hypothesized that at least $35 \%$ of practicing regional anesthesiologist participants would have used the course to guide clinical decision making since taking the training course. If the FOCUS training course has a positive effect on clinical practice, a standardized curriculum should be implemented to train resident and fellow physicians on how to incorporate this tool into their daily practice.

\section{Materials And Methods}

The Institutional Review Board approved this study at HSS. All participants who completed the FOCUS training course at HSS between March 2013 and May 2016 were eligible to participate in the study.

Eligible participants were contacted via email. As per IRB regulations, the email contained: 1) the purpose of the study, 2) an explanation that participation was voluntary and had no effect on performance evaluation or standing, 3) assurance participants could skip any question they did not want to answer and 4) contact information for questions. Each email contained a hyperlink to the survey, which was constructed and hosted on www.redcap.ctsc. med.cornell.edu/redcap_protocols/. (Supplemental 1)

Initial contact was sent on November $22^{\text {nd }}, 2016$, and reminders were sent weekly until the survey closed 12 weeks later, on February 14th 2017.

The primary outcome was the percentage of participants who have used FOCUS to guide clinical decision making since taking the training course. Secondary outcomes included 1 ) indications for FOCUS use and changes in management following basic FOCUS training, 2) the percentage of participants who feel comfortable performing the basic FOCUS exam or evaluating for pneumothorax with lung ultrasonography, 3) insight into potential benefits from a training and career perspective, and 4) perception of the limitations to basic FATE as well as the potential for course improvement.

\section{Statistical Analysis}

Assuming that $50 \%$ of the course participants used FOCUS to guide clinical decision-making, we determined that responses from 85 participants would provide $80 \%$ power at a one-sided alpha level of 0.025 to demonstrate with a one-sided binomial test that the percentage of patients who use FOCUS to guide clinical decision making is not lower than $35 \%$. Based on a previous survey, we anticipated 112 responses (i.e., a 59\% response rate).

Continuous variables were summarized as means with standard deviations or medians with $1^{\text {st }}$ and $3^{\text {rd }}$ quartiles. Categorical variables were summarized as counts and percentages. A Wilcoxon rank-sum test was used to compare time since the participant took the FOCUS course between survey responders and non-responders. A $\chi^{2}$ test was used to compare sex between survey responders and non-responders. One-sided binomial tests were used to test whether the percentage of participants that use of FOCUS to guide clinical decision-making is not lower than $35 \%$, both excluding non-responders and assuming that all nonresponders do not use FOCUS. Simple logistic regression was used to test the association between the time since the participant took the FOCUS course and the odds of using FOCUS to guide clinical decision-making, assuming that all non-responders do not use FOCUS. Multivariable logistic regression was used to test the association of time since the participant took the FOCUS course, primary specialty, and type of anesthesia practice with the odds of using FOCUS to guide clinical decision-making among responders. Statistical analyses were performed with SAS Version 9.4 (SAS Institute, Cary, NC).

\section{Results}

A total of 183 participants were eligible to participate in the course. Sixteen emails bounced back upon the first contact. After email addresses were updated, eight emails bounced back. One participant completed both the January 2015 and April 2015 courses; the survey completed for the most recent course was included in data analysis. Ninety- 
two participants total responded to the survey, of which $65(70.7 \%)$ indicated they had regional anesthesiology training or regularly practiced regional anesthesia. of the total number of respondents, 50\% (95\% CI: 40.3\%, $59.8 \%$; P-value $=0.001$ ) have used FOCUS to guide clinical decision making since taking the course. Of the regional anesthesiologists, $27(45.80 \%)$ have used FOCUS to guide clinical decision making since taking the course.

As seen in Table 1, 65 (70.7\%) of the 92 respondents reported regional anesthesia as their primary practice. At the time, they completed the FOCUS course, 38 (58.5\%), 18 (27.7\%), and $8(12.3 \%)$ of these participants were anesthesiology attendings, fellows, and residents, respectively. At the time of completing the survey, 61 (93.85\%) participants were attendings.

Participants were asked if they used FOCUS to guide clinical decision making since taking the course to which $45.80 \%$ of participants responded yes (Figure 2a). Figure $2 \mathrm{~b}$ summarizes how often FOCUS was performed, how often FOCUS was used to evaluate for pleural effusion, and how often the lung ultrasound was used to evaluate for pneumothorax. Responsesincluded 1) never,2) infrequently (once to a few times a year), 3) moderately (once to a few times a month), and 4) frequently (once to a few times a

Table 1: Demographics

\begin{tabular}{|l|c|c|}
\hline & Regional & All \\
\hline Count & $65(70.7 \%)$ & $92(100 \%)$ \\
\hline Sex & & \\
\hline Male & $46(70.8 \%)$ & $63(68.5 \%)$ \\
\hline Female & $19(29.2 \%)$ & $29(31.5 \%)$ \\
\hline Age (years) & $41 \pm 10$ & $41 \pm 11$ \\
\hline Current Title & & \\
\hline Attending & $61(93.8 \%)$ & $81(88.0 \%)$ \\
\hline Fellow & $2(3.1 \%)$ & $6(6.5 \%)$ \\
\hline Resident & $1(1.5 \%)$ & $3(3.3 \%)$ \\
\hline Title During Training Course & & \\
\hline Attending & $38(58.5 \%)$ & $50(54.3 \%)$ \\
\hline Fellow & $18(27.7 \%)$ & $20(21.7 \%)$ \\
\hline Resident & $8(12.3 \%)$ & $20(21.7 \%)$ \\
\hline Current Practice & & \\
\hline Academic & $24(36.9 \%)$ & $38(41.3 \%)$ \\
\hline Private & $12(36.9 \%)$ & $18(41.3 \%)$ \\
\hline Private with some teaching & $27(41.5 \%)$ & $29(31.5 \%)$ \\
\hline In training & $2(3.1 \%)$ & $6(6.5 \%)$ \\
\hline Anesthesiology Type & & \\
\hline General & $23(35.4 \%)$ & $39(42.4 \%)$ \\
\hline Cardiothoracic & $1(1.5 \%)$ & $5(5.4 \%)$ \\
\hline Critical care & $1(1.5 \%)$ & $3(3.3 \%)$ \\
\hline OB-anesthesia & $0(0 \%)$ & $1(1.1 \%)$ \\
\hline Pediatric & $1(1.5 \%)$ & $2(2.2 \%)$ \\
\hline Regional Anesthesia and Acute Pain & $36(55.4 \%)$ & $36(39.1 \%)$ \\
\hline Ambulatory Anesthesia & $1(1.5 \%)$ & $3(3.3 \%)$ \\
\hline Da presented as count & & \\
\hline
\end{tabular}

Data presented as count (frequency) for categorical variables and mean \pm SD for continuous variables $\mathrm{SD}=$ standard deviation; $\mathrm{OB}=$ obstetrics

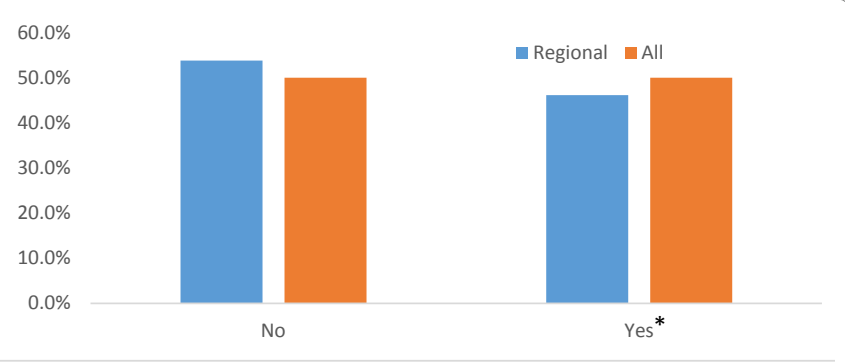

*50\% (95\% Cl: 40.3\%, 59.8\%; P-value $=0.001$

Figure 2a: Have you used FATE to guide clinical decision making since taking the course?

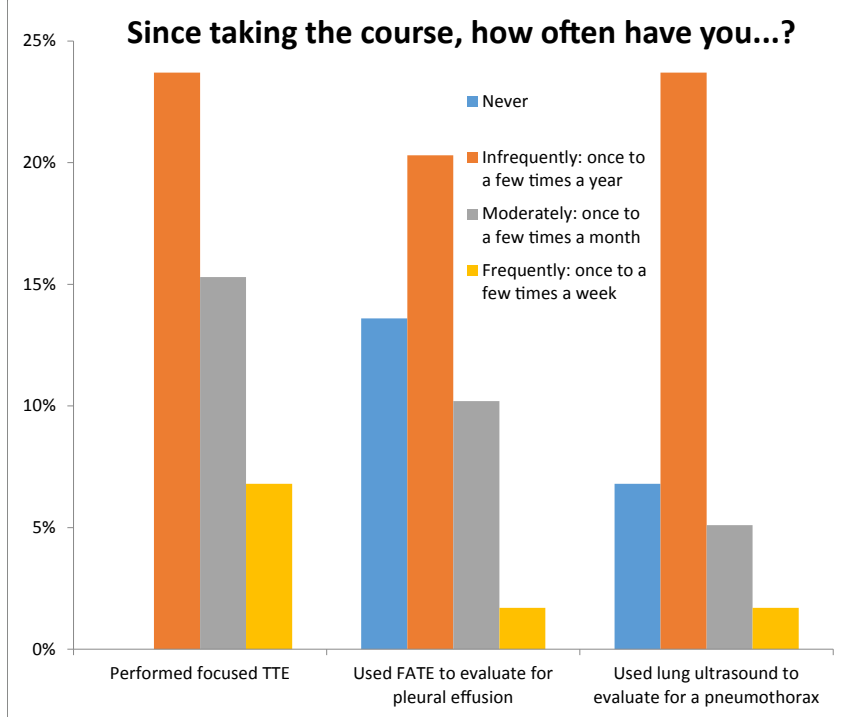

Figure 2b: Frequency of using TTE, using focused TTE to evaluate for pleural effusion, and used lung US to evaluate for pneumothorax

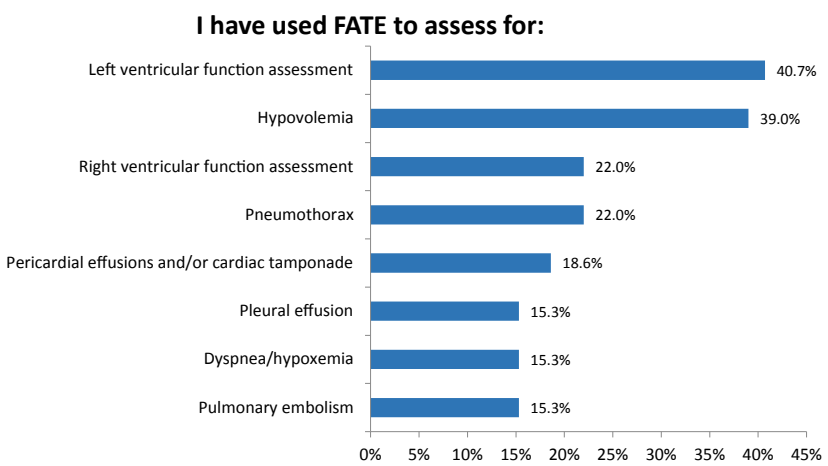

Figure 2c: FATE used to assess for...

week). Figure $1 \mathrm{c}$ depicts the clinical circumstances that regional anesthesiologists used FOCUS of which left ventricular function assessment (40.7\%) and hypovolemia (39.0\%) were most common. Participants were also asked if they used FOCUS to make changes in management in several categories, including changes in fluid management, and changes in medication management; these results are represented in Figure 2d. 


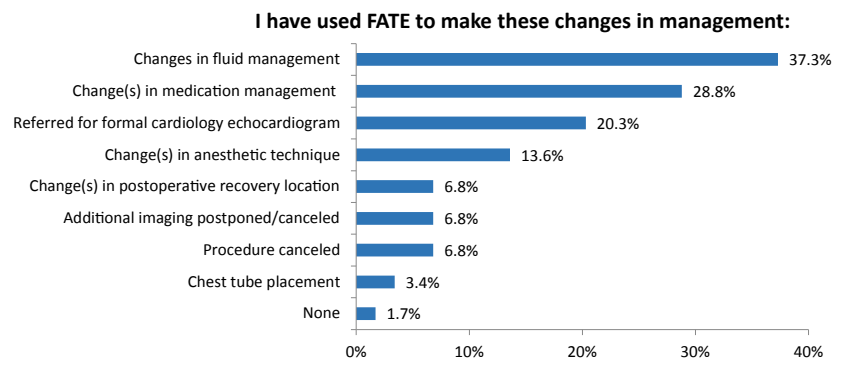

Figure 2d: FATE used for changes in management of...

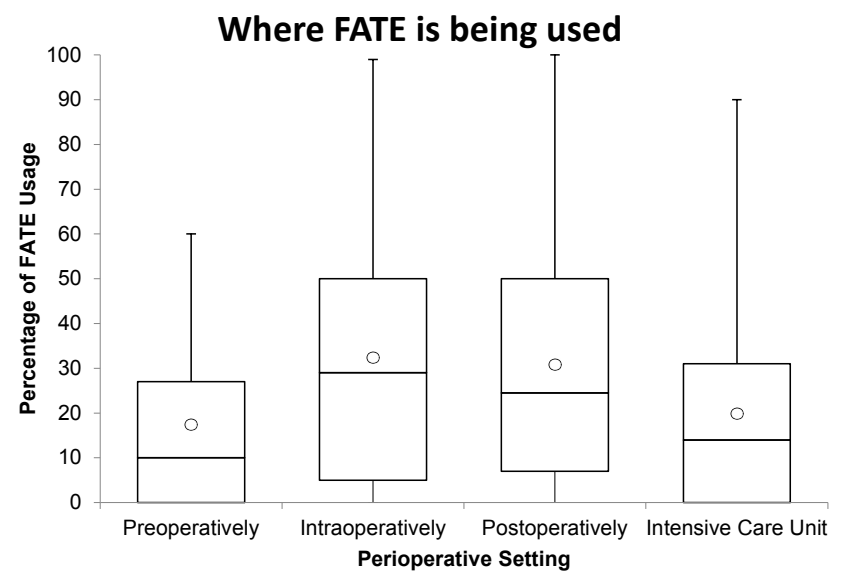

Figure 3: Where FATE is being used by Regional anesthesiologists?

Table 2: Limitations to TTE

What factors have limited your ability to implement the skills taught during the focused TTE training course?

Lack of resources, $\mathrm{n}(\%)$ $24(36.9 \%)$

Lack of opportunities, $n(\%)$

$34(52.3 \%)$

Lack of relevant clinical scenarios $28(43.1 \%)$

Currently a trainee and attendings refuse

or there is a lack of guidance

$4(6.2 \%)$

Push back from other departments

$1(1.5 \%)$

Other

$3(4.6 \%)$

Lack of comfort performing exam, $\mathrm{n}(\%)$

$20(30.8 \%)$

Concern for medical liability, $\mathrm{n}(\%)$ $11(16.9 \%)$

None, $\mathrm{n}(\%)$ $10(15.4 \%)$

$44.6 \%$ of regional anesthesiologists reported using the FOCUS skills preoperatively. $41.5 \%$ reported using the FOCUS skill intraoperatively. $41.5 \%$ reported using the FOCUS skills postoperatively, and $40.0 \%$ reports using the FOCUS skills in the Intensive Care Unit (ICU). These results are shown in Figure 3.

The limitations to the implementation of FOCUS skills are shown in Table 2. The most commonly reported limitation was lack of opportunities (52.3\%) as they relate to relevant clinical scenarios and lack of attending guidance as a resident. Twenty participants $(30.8 \%)$ responded that their ability to perform the exam was limited due to lack of comfort, while twenty-four (36.9\%) reported being unable to implement the skills due to a lack of resources. To further assess the participants' comfort level using FOCUS, participants were asked how comfortable they were performing the exam overall and how comfortable they are using the exam to evaluate for a pneumothorax (Figure 4). Their responses to both questions included not comfortable, slightly comfortable, somewhat comfortable, moderately comfortable, and extremely comfortable.

The vast majority of regional anesthesiologist respondents $(84.40 \%)$ agreed that basic FOCUS training should be a required part of anesthesia residents or fellows' curriculum (Figure 5a). Respondents were also asked if basic FOCUS training provided them with an advantage while applying in the job market and if the training affected their current practice and patient care positively (Figure $5 b)$.

\section{Discussion}

This survey-based study is one of the first to evaluate the clinical uses, indications, limitations, and benefits of FOCUS and LUS training for regional anesthesiologists. Results indicated $45.8 \%$ of regional anesthesia trained physicians utilized the FOCUS skills since completing the course. It has been utilized most frequently for assessment of left ventricular function assessment and hypovolemia, as well as to make changes in fluid and medication management.

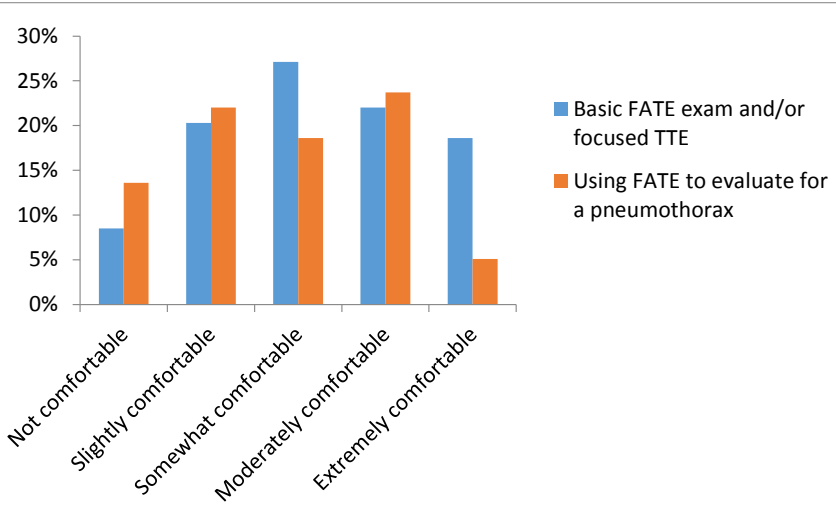

Figure 4: Comfort level with using FATE exam

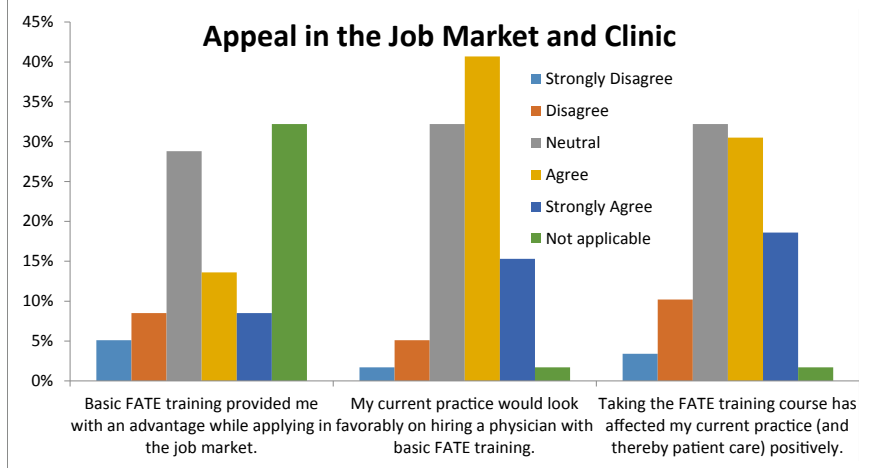

Figure 5a: Appeal in the Job Market and Clinic 


\section{Do you believe FATE training should be a required part of anesthesia residents or fellows' curriculum?}

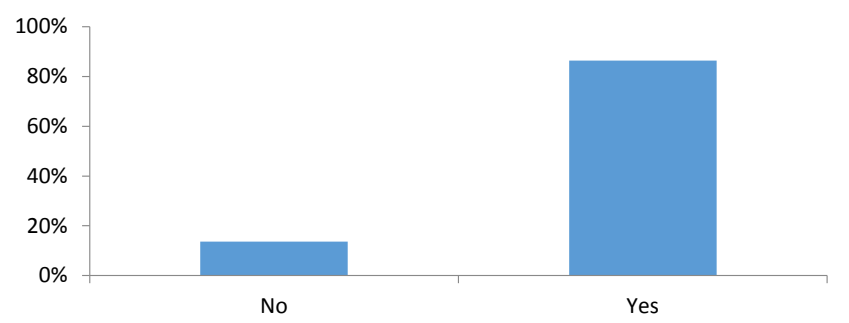

Figure 5b: Do you believe focused TTE training should be a required part of anesthesia residents or fellows' curriculum?

Within the regional anesthesia setting, the use of FOCUS has its limitations, such as lack of relevant clinical scenarios and lack of resources. Despite these limitations, nearly 85\% of survey participants felt that basic FOCUS training should be required for residents and fellows.

As POCUS, including FOCUS and LUS, becomes commonplace in the perioperative setting, it is necessary to ensure residents, fellows, and attendings are provided with appropriate and effective formal training. Regional anesthesiologists who completed such training at HSS reported an increase in the use of FOCUS and LUS for diagnostic and medical management purposes. Although several factors limited their ability to implement these acquired skills, having the training did positively affect their current practice. This evaluation of the basic FATE course demonstrates how POCUS can be used for clinical decision making for practicing regional anesthesiologists as well as identifying major barriers to implementation.

To our knowledge, this is the first study explicitly evaluating the clinical utilization of FOCUS and LUS for regional anesthesiologists. Given that POCUS has become more commonplace in regional anesthesia as a result of increased awareness in publications ${ }^{2-4}$ and more opportunities to get training through hands-on courses ${ }^{12}$, this study provides insight into the future role FOCUS and LUS and play for practicing regional anesthesiologists.

The limitations of this study include the training occurring at a single center as well as the use of a specific training course, Basic FATE, as the model for training. Therefore, generalizability of the outcome is limited as demonstrated by the increased use in the ICU setting despite few practicing critical care anesthesiologists. This particular outcome is a manifestation of trainees using these skills in ICUs under faculty supervision as well as a coverage model at HSS where anesthesiologists manage ICU patients overnight. Additionally, this study only evaluates FOCUS and LUS, while other skills such as Gastric Ultrasound ${ }^{20}$, airway US, and the focused Assessment with
Sonography for Trauma (FAST) exam $^{21}$ are also relevant to regional anesthesiologists. Future studies should pool data from a variety of POCUS courses to further identify changes in practice as well as the clinical benefits following POCUS training.

\section{Conflict of interest}

The authors have no conflicts of interest to declare.

\section{Funding}

This study was funded internally by the Department of Anesthesiology, Hospital for Special Surgery, New York, USA

\section{References}

1. Canty DJ, Royse CF. Audit of anaesthetist-performed echocardiography on perioperative management decisions for non-cardiac surgery. Br J Anaesth. 2009; 103: 352-8.

2. Haskins SC, Tanaka CY, Boublik J, et al. Focused Cardiac Ultrasound for the Regional Anesthesiologist and Pain Specialist. Reg Anesth Pain Med. 2017; 42: 632-644.

3. Haskins SC, Tsui BC, Nejim JA, et al. Lung Ultrasound for the Regional Anesthesiologist and Acute Pain Specialist. Reg Anesth Pain Med. 2017; 42: 289-298.

4. Haskins SC, Boublik J, Wu CL. Point-of-Care Ultrasound for the Regional Anesthesiologist and Pain Specialist: A Series Introduction. Reg Anesth Pain Med. 2017; 42: 281-282.

5. Zieleskiewicz L, Bouvet L, Einav S, et al. Diagnostic point-of-care ultrasound: applications in obstetric anaesthetic management. Anaesthesia. 2018; 73: 1265-1279.

6. Moore CL, Copel JA. Point-of-care ultrasonography. N Engl J Med. 2011; 364: 749-757.

7. Frankel HL, Kirkpatrick AW, Elbarbary M, et al. Guidelines for the Appropriate Use of Bedside General and Cardiac Ultrasonography in the Evaluation of Critically Ill Patients-Part I: General Ultrasonography. Crit Care Med. 2015; 43: 2479-502.

8. Levitov A, Frankel HL, Blaivas M, et al. Guidelines for the Appropriate Use of Bedside General and Cardiac Ultrasonography in the Evaluation of Critically Ill Patients-Part II: Cardiac Ultrasonography. Crit Care Med. 2016; 44: 1206-27.

9. Spencer KT, Kimura BJ, Korcarz CE, et al. Focused cardiac ultrasound: recommendations from the American Society of Echocardiography. J Am Soc Echocardiogr. 2013; 26: 567-81.

10. Fagley RE, Haney MF, Beraud AS, et al. Society of Critical Care Anesthesiologists. Critical Care Basic Ultrasound Learning Goals for American Anesthesiology Critical Care Trainees: Recommendations from an Expert Group. Anesth Analg. 2015; 120: 1041-53.

11. Singh Y, Tissot C, Fraga MV, et al. International evidence-based guidelines on Point of Care Ultrasound (POCUS) for critically ill neonates and children issued by the POCUS Working Group of the European Society of Paediatric and Neonatal Intensive Care (ESPNIC). Crit Care. 2020; 24: 65.

12. https://www.asra.com/page/2606/introduction-to-perioperativepoint-of-care-ultrasound

13. Tanzola RC, Walsh S, Hopman WM, et al. Brief report: Focused transthoracic echocardiography training in a cohort of Canadian anesthesiology residents: a pilot study. Can J Anaesth. 2013; 60: 32-7.

14. Cowie B. Three years' experience of focused cardiovascular ultrasound in the perioperative period. Anaesthesia. 2011; 66: 268-73. 
15. Haskins SC, Zhao J, Nejim JA, et al. Evaluation of Postgraduates Following Implementation of a Focus Assessed Transthoracic Echocardiography (FATE) Training Course-A Pilot Study. J Anesth Clin Res. 2017; 8: 763.

16. Ramsingh D, Rinehart J, Kain Z, et al. Impact assessment of perioperative point-of-care ultrasound training on anesthesiology residents. Anesthesiology. 2015; 123: 670-82.

17. Rempell JS, Saldana F, DiSalvo D, et al. Pilot Point-of-Care Ultrasound Curriculum at Harvard Medical School: Early Experience. West J Emerg Med. 2016; 17: 734-740.

18. Jensen MB, Sloth E, Larsen KM, et al. Transthoracic echocardiography for cardiopulmonary monitoring in intensive care. Eur J Anaesthesiol. 2004; 21: 700-707.

19. Neal JM, Liguori GA, Hargett MJ. The training and careers of regional anesthesiology and acute pain medicine fellows, 2013. Reg Anesth Pain Med. 2015; 40: 218-22.

20. Haskins SC, Kruisselbrink R, Boublik J, et al. Gastric Ultrasound for the Regional Anesthesiologist and Pain Specialist. Reg Anesth Pain Med. 2018; 43: 689-698.

21. Haskins SC, Desai NA, Fields KG, et al. Diagnosis of Intraabdominal Fluid Extravasation After Hip Arthroscopy with Point-of-Care Ultrasonography Can Identify Patients at an Increased Risk for Postoperative Pain. Anesth Analg. 2017; 124: 791-799. 